\title{
Bubbles in Stochastic Economies: Can They Cure Overaccumulation of Capital?
}

\author{
Mathias Binswanger \\ Received September 3, 2003; revised version received September 29, 2004 \\ Published online: February 14, 2005 \\ (C) Springer-Verlag 2005
}

It is well known that bubbles possess the potential to increase economic welfare due to a reduction of capital accumulation in deterministic overlapping generations economies that are in a dynamically inefficient state. However, actual economies are stochastic, where the concept of dynamic efficiency has turned out to be a complex issue. This paper contributes in two ways. First, the model presented in this paper establishes that dynamic inefficiency is not a necessary condition for deterministic bubbles in a stochastic economy. Second, a simulation shows that although bubbles cannot persist in the stochastic steady state, they can still cure overaccumulation of capital for a time long enough to cover agents' relevant time horizon.

Keywords: bubbles, dynamic efficiency, capital overaccumulation, stochastic OLG model.

JEL Classification: D61, E44.

\section{Introduction}

Research on bubbles in deterministic overlapping generations models has shown that they are able to move a dynamically inefficient economy towards dynamic efficiency through a reduction of capital accumulation (Cass, 1972; Tirole, 1985; Weil, 1987; Blanchard and Fischer, 1989, provide an overview of the main arguments). However, it is not clear so far, whether the potential to restore dynamic efficiency by speculative bubbles is of any relevance to actual economies. In this paper, we discuss this question in relation to a simple stochastic overlapping generations economy, where, as is also the case in reality, the return to capital is 
uncertain. Considering a stochastic economy is important because recent theoretical and empirical results may lead to the conclusion that the welfare increasing potential of bubbles due to prevention of capital overaccumulation, that has been established for deterministic economies, is irrelevant in stochastic production economies. On the one hand, recently developed criteria for the assessment of dynamic inefficiency in stochastic economies indicate that the US economy as well as the economies in other industrialized countries are dynamically efficient (Abel et al., 1989; Barbie et al., 2004; Cohen et al., 1995), which seems to rule out the existence of bubbles. And on the other hand, theoretical models imply that, even if economies were dynamically inefficient, the resulting bubble equilibria would eventually be unstable and, therefore, bubbles would no longer be able to restore dynamic efficiency (Bertocchi, 1994).

The main objective of the paper is to show that the existence of bubbles cannot generally be ruled out in stochastic economies and that under certain conditions bubbles can still cure overaccumulation of capital. Starting with a general overlapping generations model that considers stochastic bubbles in a stochastic economy we show that risk premia for investment in real capital as well as for investment in bubbles play a crucial role in the resulting equilibria. Only if agents are risk neutral the expected returns to both investments will be equal. However, dynamic efficiency cannot be evaluated properly in this general model as this is possible only for bubbles that exist for an infinite future. Analyzing a more restricted model of a stochastic economy with a never collapsing bubble reveals that under these circumstances dynamic inefficiency is not a necessary condition for the existence of stationary bubbles.

But there remains the argument that stationary states in a stochastic economy with a bubble are not stable. However, in a simulation exercise we show that stationary bubbles can still exist over long time periods, without exploding or converging towards zero, although not for an infinite future. But, as we will argue, an infinitive future is not a relevant time scale for actual economic decision making and theoretical results based on the assumption of an infinite future may not be directly applicable to actually existing economies. Therefore, bubbles still possess a welfare increasing potential in stochastic economies if these economies are in a dynamically inefficient state.

The paper is organized as follows. In Sect. 2, we present a short overview of the existing literature concerning the welfare increasing 
potential of bubbles in dynamically inefficient economies. In Sect. 3, we present a simple overlapping generations economy, where we consider the most general case of a stochastic bubble (bubbles that may collapse in each period) in a stochastic production economy. Section 4 presents a criterion for assessing dynamic inefficiency in stochastic economies proposed by Zilcha (1991) and applies it to a special version of the model developed in Sect. 3, where bubbles never collapse. Section 4 also demonstrates how bubbles are able to cure stochastic economies from capital overaccumulation and how the conditions for the existence of bubbles are related to dynamic efficiency. Section 5 presents a simulation of a bubble economy with logarithmic utility and a Cobb-Douglas production function. Section 6 outlines some implications of this simulation for empirical research concerning dynamic efficiency and the possible existence of bubbles. Section 7 concludes and highlights the main results.

\section{A Brief Survey of the Literature on Dynamic Inefficiency and Bubbles}

Research on bubbles has shown that, provided that agents have rational expectations, bubbles can only exist in deterministic intertemporal economies if the number of periods is infinite but agents' time horizon is finite. These conditions are fulfilled in an overlapping generations economy where each period the old generation of agents leaves the economy and a new generation of agents enters the economy. However, a further necessary condition for bubbles to exist in a deterministic overlapping generations economy is that the economy is in a dynamically inefficient state due to overaccumulation of real capital (see Cass, 1972; Tirole, 1985; Weil, 1987). An important implication of this result is the potential of bubbles to move the economy towards dynamic efficiency as they reduce capital accumulation. Therefore, sustainable bubbles can be considered to be beneficial as they increase the potential level of consumption for everybody. In this respect, there is a tight connection between sustainable bubbles and sustainable debt Ponzi games (infinite government debt rollover) as the latter are also able to reduce capital accumulation in an overlapping generations model as shown in Tirole (1985).

Traditionally, overlapping generations models including bubbles consider an economy where individuals have the possibility to invest either in real capital or in a bubble. However, it has turned out that the conditions under which bubbles may exist in these models crucially depend on 
whether the economy is deterministic or stochastic. Tirole (1985) has established conditions under which a deterministic bubble may exist in a deterministic economy without uncertainty about the return to real capital. This model has been further developed by Weil (1987) who, in a similar way, examines stochastic bubbles in a deterministic economy. These models essentially show that dynamic inefficiency, which is characterized by a growth rate of the economy above the return to capital (which equals the riskless interest rate in a deterministic economy), is a necessary but in the case of stochastic bubbles not sufficient condition for the possible existence of bubbles.

However, actual economies are stochastic and dynamic efficiency in stochastic production economies has turned out to be a subtle issue (see Abel et al., 1989; Barbie et al., 2001; 2004; Bertocchi, 1991; 1994; Bertocchi and Kehagias, 1995; Blanchard and Weil, 2001; Zilcha, 1990; 1991). ${ }^{1}$ First of all, Abel et al. (1989) showed that a simple comparison of the riskless rate and the growth rate of the economy cannot be used to evaluate dynamic efficiency in a stochastic economy as it neglects the impact of risk. Therefore, economies may still be dynamically efficient even if the average riskless rate is below the growth rate of the economy.

Abel et al. (1989) developed a different condition for dynamic efficiency from maximization of a social planner's welfare function with predetermined utility functions stating that an economy is dynamically efficient as long as gross profits exceed gross investment in every year (net cash flow criterion). However, being a sufficient condition for dynamic efficiency, the net cash flow criterion is often not conclusive in particular models (such as the one presented in this paper), where the return to capital fluctuates and sometimes is above and other times is below the growth rate of the economy (Blanchard and Weil, 2001). Subsequently, Zilcha (1991) has derived a necessary and sufficient con-

1 Abel et al. (1989), Bertocchi and Kehagias (1995), Blanchard and Weil (2001), and Barbie et al. (2001), all emphasize that the concepts of dynamic efficiency and Pareto optimality must be distinguished in stochastic economies. Welfare evaluations are more complex than in deterministic economies because inefficiency has two potential sources: capital overaccumulation and imperfect risk-sharing between generations, which can both be cured by sustainable Ponzi games or deterministic bubbles. Pareto improvements in dynamically efficient economies are still possible because of missing insurance markets. In this paper, we will concentrate on the issue of overaccumulation, which is related to dynamic inefficiency and neglect the issue of intergenerational risk sharing. 
dition for dynamic efficiency in stationary stochastic economies that, similar to deterministic economies, compares the expected return to capital to the growth rate of the economy, and which we will also use in this paper. Both, the net cash flow criterion and the criterion developed by Zilcha appear to indicate that the US economy as well as the economies in other industrialized countries are dynamically efficient (Abel et al., 1989; Barbie et al., 2001; 2004).

Even if dynamic efficiency can be assessed in a stochastic production economy, there remains the question under what conditions bubbles can exist and whether they can actually increase welfare. So far, there are very few contributions that deal with these issues. Bertocchi (1991) examines deterministic bubbles, which never burst, in a stochastic economy showing that, in this case, there is no direct link between the existence of bubbles and dynamic efficiency. And Bertocchi (1994) shows that the conditions to ensure the existence of stationary states with rolled-over debt (which can be interpreted as a deterministic bubble) are very restrictive. Although bubbles may exist in stochastic economies, the resulting equilibria appear to be unstable and the bubble either explodes or converges towards zero. Therefore, bubbles cannot restore dynamic efficiency forever as in a deterministic economy and the welfare increasing potential of bubbles, that has been established for deterministic economies, may not carry over to stochastic economies.

\section{Description of a Stochastic Economy with a Stochastic Bubble}

The following model is based on Diamond (1965), who used the overlapping generations model to describe a neoclassical production economy. The economy consists of identical two-period-lived agents, so that, at any point of time, there coexist two generations, the young and the old. An agent of generation $t$ consumes $c_{1 t}$ when young and $c_{2 t}$ when old. The population born at time $t$ and working during period $t$ is denoted $N_{t}$ and grows at the rate $n$. Therefore, $N_{t+1}=N_{t}(1+n)=N_{0}(1+n)^{t+1}$. Agents work only in the first period of their lives and supply inelastically one unit of labor earning a real wage of $w_{t}$. Output is given by the neoclassical constant returns to scale production function $Y_{t}=A_{t} F\left(K_{t}, N_{t}\right)$, where $A_{t}$ denotes the level of productivity. Output is produced by firms, who maximize profits, hire labor and invest in capital to the point where $w_{t}$ and the rental rate on capital equal the marginal product with respect to labor and capital and, therefore, $w_{t}=A_{t}\left[f\left(k_{t}\right)-f^{\prime}\left(k_{t}\right) k_{t}\right]$. Output per worker 
$Y_{t} / N_{t}$, denoted $y_{t}$, is given by the production function $y_{t}=A_{t} f\left(k_{t}\right)$, where $k_{t}$ is the capital-labor ratio. Further, to simplify the following analysis, it is assumed that $A_{t} F\left(K_{t}, N_{t}\right)$ is a net production function ${ }^{2}$ with depreciation already accounted for, that is twice differentiable, exhibits positive and diminishing marginal products with respect to $N_{t}$ and $K_{t}$ and satisfies the Inada conditions. Technological progress is not considered here but we could easily introduce labor-augmenting technological change into the model without altering the main thrust of the arguments.

In a fashion similar to Tirole (1985) and Weil (1987) we modify the original Diamond model by enlarging the investment possibilities of each agent. Agents can invest either in real capital or in bubbles, which in period $t$ can be bought at a price $P_{t}$. These bubbles may evolve on an intrinsically useless asset (pure bubbles) or on assets with a market fundamental, such as aggregate equities, whose fundamental value may also change. In the latter case, $P_{t}$ should actually be interpreted as the bubble component of the asset's total price. Tirole (1985) shows that the results derived for bubbles on intrinsically useless assets can be generalized to assets paying a dividend as long as dividends grow at a slower rate than the economy as we will assume throughout the paper. In this case, the price of the asset goes asymptotically towards the value of the bubble component of the asset price. Therefore, we neglect the fundamental value and make the simplifying assumption that also in the case when the bubble evolves on a dividend paying asset the value of the asset equals the value of the bubble. We further assume that the asset, on which the bubble evolves, is in fixed supply, $M$, with $m$ denoting the supply of the bubble per worker.

The formalization of a stochastic bubble follows Blanchard (1979), and Blanchard and Watson (1982). A bubble that exists in period $t$ is supposed to survive with a probability $q$ in period $t+1$ and, consequently, the bubble collapses with probability $1-q$. The price of the bubble, $P_{t+1}$, in period $t+1$ is governed by a Markov process where, as long as the bubble survives,

$$
P_{t+1}=\left(1+r_{t}\right) q^{-1} P_{t}+\epsilon_{t+1}
$$

2 The net production function $A_{t} f\left(k_{t}\right)$ is defined as $A_{t} f\left(k_{t}\right)=A_{t} g\left(k_{t}\right)-\delta k_{t}$, where $A_{t} g\left(k_{t}\right)$ is the gross production function and $\delta$ stands for the depreciation rate. 
with $r_{t}$ denoting the required rate of return at time $t, \epsilon_{t}$ being a stochastic term with $E_{t}\left(\epsilon_{t+1}\right)=0$, and $P_{t}, P_{t+1}>0$. Provided that $P_{t}>0$ and that investors are guided by rational expectations, the expected return to the bubble conditional on information available at time $t$ is therefore

$$
\frac{E_{t}\left(P_{t+1}\right)}{P_{t}}=\left(1+r_{t}\right) q^{-1} .
$$

In order to keep the model as simple as possible we will also model the stochastic production economy by using fixed probabilities. We assume that $y_{t}$ may take on different values in each period through the level of productivity $A_{t}$, which randomly fluctuates between two states of nature $A_{H}$ and $A_{L}$. The level of productivity follows an i.i.d. process that is stationary and given by

$$
\begin{array}{ll}
A_{t+1}=A_{H} & \text { with probability } z, \\
A_{t+1}=A_{L} & \text { with probability } 1-z,
\end{array}
$$

where $A_{H}>A_{L}>0$. The stochastic process governing production in our overlapping generations model is identical to the stochastic process used in the overlapping generations economy presented in Bertocchi (1994), which makes our results easily comparable to the results presented there.

In order to make the model more tractable we will use the notation $A_{H}=(1+h) A_{L}$ where $h>0$. In this case the expected value of $A_{t+1}$ in each period is

$$
E_{t}\left(A_{t+1}\right)=(1+h z) A_{L} .
$$

An investor faces the constraints

$$
\begin{gathered}
w_{t}=c_{1 t}+i_{t}+P_{t} m_{t}, \\
c_{2 t}=i_{t}\left(1+A_{t+1} f^{\prime}\left(k_{t+1}\right)\right)+P_{t+1} m_{t},
\end{gathered}
$$

where $i_{t}$ stands for investment in real capital. Assuming that utility is time separable, a young born at time $t$, chooses $c_{1 t}, c_{2 t}$, and $m_{t}$ to maximize the following expected utility function:

$$
\max E_{t}\left[u\left(c_{1 t}\right)+\beta u\left(c_{2 t}\right)\right]
$$


subject to constraints (3) and (4). $E_{t}$ stands for the expected value conditional on information available at $t, \beta=(1+\Phi)^{-1}$ is the subjective discount factor with $\Phi$ being the subjective rate of time preference $(\Phi>0)$. Using (3) to substitute for $i_{t}$ yields the following first-order conditions

$$
\begin{gathered}
E_{t}\left[u^{\prime}\left(c_{1 t}\right)-\beta\left[1+A_{t+1} f^{\prime}\left(k_{t+1}\right)\right] u^{\prime}\left(c_{2 t}\right)\right]=0, \\
E_{t}\left[u^{\prime}\left(c_{1 t}\right)-\beta \frac{q P_{t+1}}{P_{t}} u^{\prime}\left(c_{2 t}\right)\right]=0 .
\end{gathered}
$$

The first-order conditions (5) and (6) imply that for risk neutral agents, for which $u^{\prime}()$ is a constant

$$
\frac{q P_{t+1}}{P_{t}}=1+E_{t}\left[A_{t+1} f^{\prime}\left(k_{t+1}\right)\right]=1+(1+h z) A_{L} f^{\prime}\left(k_{t+1}\right),
$$

which equates the expected return to the bubble to the expected return to real capital.

Condition (7) only holds for risk neutral agents and we can make no general statement, whether, for risk averse agents, the expected return to the bubble must be larger or smaller than the expected return to real capital. However, we can look at the two limiting cases where, first, the bubble is deterministic and, second, the return to capital is deterministic. In the first case, $\epsilon_{t}=0$ and $q=1$, as the bubble will survive with certainty in every future period. From (5) and (6), it follows:

$$
\begin{aligned}
\frac{P_{t+1}}{P_{t}}= & \frac{E_{t}\left[u^{\prime}\left(c_{2 t}\right)\left(1+A_{t+1} f^{\prime}\left(k_{t+1}\right)\right]\right.}{E_{t}\left[u^{\prime}\left(c_{2 t}\right)\right]} \\
& =\frac{E_{t}\left[u^{\prime}\left(c_{2 t}\right)\right] E_{t}\left[\left(1+A_{t+1} f^{\prime}\left(k_{t+1}\right)\right]+\operatorname{COV}\left[\left(u^{\prime}\left(c_{2 t}\right),\left(A_{t+1} f^{\prime}\left(k_{t+1}\right)\right]\right.\right.\right.}{E_{t}\left[u^{\prime}\left(c_{2 t}\right)\right]} \\
& =E_{t}\left[\left(1+A_{t+1} f^{\prime}\left(k_{t+1}\right)\right]+\frac{\operatorname{COV}\left[\left(u^{\prime}\left(c_{2 t}\right),\left(A_{t+1} f^{\prime}\left(k_{t+1}\right)\right]\right.\right.}{E_{t}\left[u^{\prime}\left(c_{2 t}\right)\right]}\right.
\end{aligned}
$$

As $\operatorname{COV}\left[\left(u^{\prime}\left(c_{2 t}\right),\left(A_{t+1} f^{\prime}\left(k_{t+1}\right)\right]\right.\right.$ is always negative if $u$ is strictly concave, condition (8) implies that investors will demand a risk premium on investment in real capital and that, therefore, the required return to capital 
must be higher than the return to the bubble with equality only holding for risk neutral investors ${ }^{3}$

$$
\frac{P_{t+1}}{P_{t}} \leq E_{t}\left[\left(1+A_{L} f^{\prime}\left(k_{t+1}\right)\right]\right.
$$

In the second case, where the economy is deterministic and, for example, $z=0$, conditions (5) and (6) imply

$$
\frac{q P_{t+1}}{P_{t}}>1+A_{L} f^{\prime}\left(k_{t+1}\right) \text {. }
$$

Risk averse investors demand a risk premium on the bubble, and the expected return to the bubble must be higher than the return to real capital, as has already been shown in Weil $(1987, \text { p. 10) })^{5}$.

Equilibrium on the market for the bubble (or the asset on which the bubble evolves) requires that

$$
m_{t}=\frac{M}{N_{t}}
$$

(see Weil, 1987, p. 6). Equilibrium on the market for real capital requires that

3 Condition (9) is very similar to condition (6) in Bertocchi (1991, p. 119) who also examines a deterministic bubble in a stochastic economy.

4 From conditions (5) and (6), it follows that

$$
\frac{q P_{t+1}}{P_{t}}=\left[1+A_{L} f^{\prime}\left(k_{t+1}\right)\right]\left[q+(1-q) \frac{u^{\prime}\left(c_{2 t}^{-}\right)}{u^{\prime}\left(c_{2 t}^{+}\right)}\right] \geq 1+A_{L} f^{\prime}\left(k_{t+1}\right),
$$

where

$$
\begin{aligned}
& c_{2 t}^{+}=i_{t}\left(1+A_{L} f^{\prime}\left(k_{t+1}\right)\right)+P_{t+1} m, \\
& c_{2 t}^{-}=i_{t}\left(1+A_{L} f^{\prime}\left(k_{t+1}\right)\right)
\end{aligned}
$$

showing that agents demand a risk premium on the bubble if $u$ is increasing in $c$ and strictly concave. The term $u^{\prime}\left(c_{2 t}^{-}\right) / u^{\prime}\left(c_{2 t}^{+}\right)$generally increases with risk aversion.

5 For a stationary bubble (9) implies $q(1+n) \geq 1+A_{L} f^{\prime}(k)$, which is the necessary condition for a stationary stochastic bubble to exist in a deterministic economy (Weil, 1987, p. 13). 


$$
k_{t+1}=(1+n)^{-1} i_{t},
$$

where

$$
i_{t}=i\left[w\left(A_{t} k_{t}\right), E_{t}\left(A_{t+1} f^{\prime}\left(k_{t+1}\right)\right] .\right.
$$

Letting $b_{t}=P_{t} m_{t}$ denote the per capita value of the bubble, the saving of a young is $s_{t}=i_{t}+b_{t}$. At time $t$ the savings of the young depend on their labor income $w$, which is a function of the product of $A_{t}$ and $k_{t}$, and on the expected rate of return to savings, which is determined by the expected value of the product of $A_{t+1}$ and $f^{\prime}\left(k_{t+1}\right)$ and the expected value of the bubble. A rational expectations equilibrium of this economy is a stochastic sequence $\left\{k_{t}, b_{t}\right\}_{t=0}^{\infty}$ that satisfies the first-order conditions (5)-(6) and the individual and economy-wide constraints (Abel et al., 1989). A stationary equilibrium (a stationary state) of $\left\{k_{t}, b_{t}\right\}_{t=0}^{\infty}$ is characterized by a stationary distribution (see Bertocchi, 1994). ${ }^{6}$

\section{Existence of Bubbles and Dynamic Efficiency in a Stationary Stochastic Economy}

In this section, we will relate conditions for the existence of bubbles in stochastic economies to the concept of dynamic efficiency. We want to find out whether dynamic efficiency is also a necessary condition for bubbles to exist in a stationary stochastic economy as it is the case in a stationary deterministic economy (Tirole, 1985; Weil, 1987). Our analysis is based on the methodology proposed by Zilcha (1990; 1991), who provides a complete characterization of dynamic efficiency without

6 It still remains an open question how stationary equilibria of a stochastic bubble in a stochastic economy can be characterized in a complete way. In case of a deterministic economy the resulting stationary state exhibits saddelpoint stability as shown for a deterministic bubble (Tirole, 1985, p. 1505) and for a stochastic bubble (Weil, 1987, pp. 15-17). 
assuming particular preferences. ${ }^{7}$ But we have to take care of the fact that Zilcha's criterion is only applicable to the examination of a bubble that is expected to exist for an infinite period of time, where $q=1$. Otherwise Birkhoffs ergodic theorem, on which the criterion is based, cannot be applied (see Footnote 11). Therefore, we restrict our analysis to never collapsing, deterministic bubbles, for which the risk premium demanded on the bubble must be zero, as indicated by (8), and where $\epsilon_{t}=0$ for all $t$.

The following definitions are necessary in order to evaluate dynamic efficiency in a stationary stochastic economy. In our specific model of such an economy the first-period consumption $c_{1 t}$ of an investor born at date $t$ depends on the realizations of the level of productivity $\left(\ldots A_{0}, \ldots, A_{t}\right)$ until date $t$, where $A_{t} \in\left\{A_{L}, A_{H}\right\}$ denotes the realization at each point of time. His second period consumption $c_{2 t}$ depends on the realizations $\left(\ldots A_{0}, \ldots, A_{t+1}\right)$ till date $t+1$. The capital stock $k_{t+1}$ depends on the realizations $\left(\ldots A_{0}, \ldots, A_{t}\right)$ until date $t$ as it is determined by $i_{t}$.

Given a feasible allocation from an initial capital stock $k_{0}$, let $\Omega$ be the set of all doubly infinite sequences of random shocks $A$, i.e., A $\in\left\{A_{L}, A_{H}\right\}^{\infty}$. Let us define a shift operator $T, T: \Omega \rightarrow \Omega$ by $T A_{t}=A_{t+1}$ for all $t, T^{-1} A_{t}=A_{t-1}$ for all $t$. Because $A_{t}$ follows an i.i.d. process in our model, $T$ is measure preserving and ergodic. Furthermore, $T f$ is defined by $T f(A)=f(T A)$ a.s. (almost surely), where $f$ is a nonnegative function of $A{ }^{8}$ Using these definitions allows us to write the marginal product in every period $t^{9}$

$$
r_{t}(A)=A_{t} f^{\prime}\left(k_{t}(A)\right) \quad \text { a.s. }
$$

7 Zilcha makes one additional assumption about the elasticities of the production function and the marginal product (Zilcha, 1991, p. 5). There exist four constants $0<m_{1}, m_{2}, m_{3}, m_{4}<\infty$ such that for all $k>0$ and $A_{L}$ and $A_{H}$,

$$
m_{1}<\frac{k A_{t} f^{\prime}(k)}{A_{t} f(k)}<m_{2}, \quad m_{3}<\frac{-k A_{t} f^{\prime \prime}(k)}{A_{t} f^{\prime}(k)}<m_{4} .
$$

We will make the same assumption when applying Zilcha's efficiency criterion.

8 For further details, see Zilcha (1991, p. 4).

9 Zilcha (1991) defines the marginal product for the more general case of any kind of stochastic shock while here we restrict our model to multiplicative shocks. 
Analogously to a deterministic economy, where the focus is usually on steady states, we consider stationary production-consumption allocations in the stochastic economy. Consumption is a stationary process if at every date $t, c_{1 t}(A)=c_{1}\left(T^{t} A\right)$ a.s. for $t \geq 0, c_{2 t}(A)=c_{2}\left(T^{t} A\right)$ a.s. for $t \geq-1$. If the consumption over time $\left\{c_{1 t}, c_{2 t}\right\}_{t=0}^{\infty}$ is a stationary stochastic process the capital stock $\left\{k_{t}\right\}_{t=0}^{\infty}$ is also a stationary stochastic process with $k_{t}(A)=k\left(T^{t-1} A\right)$ a.s. for all $t \geq 0$. The marginal products of capital are also a stationary stochastic process $\left\{r\left(T^{t} A\right)\right\}_{t=0}^{\infty}$ where $r(A)$ is defined as (Zilcha, 1991, p. 5)

$$
r(A)=A_{0} f^{\prime}\left(k\left(T^{-1} A\right)\right) \quad \text { a.s. }
$$

The following dynamic efficiency criterion (15) uses the return to real capital as defined in (14). It states that a bubbleless stationary stochastic economy starting from an initial capital stock $k_{0}^{d}$ is inefficient if and only if (Zilcha, 1991, Theorem 1, p. 8)

$$
\log (1+n)>E\left[\log \left(1+r^{d}(A)\right)\right]^{10},
$$

where $r^{d}(A)$ is the return to real capital defined in (14) in the bubbleless stochastic economy. The criterion is built on the assumption that the probability distribution of future states is known to investors in each generation.

10 The criterion (15) follows from an extension of Cass' (1972) general inefficiency criterion that was developed for deterministic economies. It is formulated in logarithms which makes it possible to apply Birkhoff's ergodic theorem as the logarithm of an ergodic process is also an ergodic process (Breiman, 1968, Proposition 6.31). In this case, the expected value of $\log \left(1+r^{d}(A)\right)$ can be derived from

$$
\frac{1}{N} \sum_{t=0}^{N-1} \log \left(1+r^{d}\left(T^{t} A\right)\right) \rightarrow E\left[\log \left(1+r^{d}(A)\right)\right] \quad \text { as } \quad N \rightarrow \infty \quad \text { a.s. }
$$

(Zilcha, 1991, proof of theorem 1, p. 12).

Moreover, the efficiency criterion is built on the concept of stochastic dominance. Strictly speaking (15) only establishes dynamic inefficiency with respect to first degree stochastic dominance, which Zilcha labels type-I inefficiency. Condition (15) also establishes dynamic inefficiency with respect to second degree stochastic dominance (type-II inefficiency) if the utility $u$ is predetermined and stricly concave. 
We now consider an economy with a stationary bubble. In the first period, the economy starts with a capital stock $k_{0}=k_{0}^{\mathrm{d}}-b_{0}\left(k_{0}^{\mathrm{d}}>b_{0}\right)$ but otherwise it is identical to the bubbleless stochastic economy that starts with capital stock $k_{0}^{\mathrm{d}}$ as $A_{t}$ is governed by the same stationary stochastic process in both economies. Stationary stochastic allocations including a bubble require that the bubble $\left\{b_{t}\right\}_{t=0}^{\infty}$ itself is also a stationary stochastic process, implying that the bubble does neither converge to zero nor does it explode as $t \rightarrow \infty$. This will be the case if at every date $t, b_{t}(A)=b\left(T^{t} A\right)$ a.s.

We assume that the existence of a stationary bubble is always associated with a decrease in the capital stock relative to the stationary stochastic bubbleless Diamond economy (a restriction on tastes and technologies). ${ }^{11}$ From this assumption it is obvious that

$$
E\left[\log \left(1+\left(r^{d}(A)\right)\right]<E[\log (1+(r(A))]\right.
$$

because of the diminishing marginal returns with resepct to capital. Condition (16) implies that the expected return to capital must be higher in a stochastic economy with a bubble than in a bubbleless stochastic economy. Furthermore, Jensen's inequality implies that

$$
\log \left[E\left(1+r^{d}(A)\right)\right]>E\left[\log \left(1+\left(r^{d}(A)\right)\right] .\right.
$$

Condition (17) shows that the condition for dynamic inefficiency in a bubbleless economy (15) is less stringent than $n>E\left(r^{d}(A)\right)$, which would be the generalization of the condition for deterministic economies $n>r^{d}$.

Combining the condition for dynamic inefficiency (15) with condition (9) resulting from the utility maximization of risk averse investors allows us to formulate the following proposition, which is the central result of this section:

Proposition 1: In a stochastic economy, where the return to capital follows an ergodic stochastic process, dynamic inefficiency is not a necessary condition for the existence of a stationary equilibrium with a deterministic bubble.

11 This assumption is standard in the literature on bubbles and corresponds to assumption A in Weil (1987, p. 12). 
Proof: Inequality (9) can be rewritten as

$$
\frac{b_{t+1}}{b_{t}}(1+n) \leq E_{t}\left[\left(1+A_{t+1} f^{\prime}\left(k_{t+1}\right)\right]=E_{t}\left[\left(1+r_{t+1}(A)\right)\right]\right.
$$

or formulated in logarithms

$$
\log \left(b_{t+1}\right)-\log \left(b_{t}\right)+\log (1+n) \leq \log \left[E_{t}\left(1+r_{t+1}(A)\right)\right] .
$$

Since $b_{t}$ and $r_{t}$ are stationary, it follows from Birkhoff's ergodic theorem that

$\frac{1}{N} \sum_{t=0}^{N-1} \log \left[b\left(T^{t+1} A\right)\right]=\frac{1}{N} \sum_{t=0}^{N-1} \log \left[b\left(T^{t} A\right)\right] \rightarrow E[b(A)]$ as $N \rightarrow \infty$ a.s.

and

$\frac{1}{N} \sum_{t=0}^{N-1} \log \left[E_{t}\left(1+r\left(T^{t+1} A\right)\right)\right] \rightarrow \log [E(1+r(A))] \quad$ as $\quad N \rightarrow \infty \quad$ a.s.

Using (19) and (20), we can take expectations on both sides of (18a) and obtain

$$
\log (1+n) \leq \log [E(1+r(A))] .
$$

Let us combine (21) with the inefficiency condition in the bubbleless economy (15). Consistency of these conditions leads to the requirement that

$$
E\left[\log \left(1+r^{d}(A)\right)\right]<\log (1+n) \leq \log [E(1+r(A))] .
$$

The requirement (22) shows that a stationary bubble may still exist if condition (15) is not fulfilled, i.e., $\log (1+n) \leq E\left[\log \left(1+r^{d}(A)\right)\right]$, because risk averse investors require that the rate of return to capital is higher than the return to the bubble, which equals the growth rate in a stationary economy. Therefore (15) cannot be a necessary condition for the existence of a stationary bubble.

Proposition 1 can be compared to the conditions for the existence of bubbles in deterministic economies derived in Tirole (1985, p. 1504) and 
in Weil (1987, p. 13). In a stochastic economy a stationary deterministic bubble may even exist in a dynamically efficient economy where $\log (1+n)<E\left[\log \left(1+r^{d}(A)\right)\right]$ as the required return to the bubble is lower than the expected return to capital. Generally, the conditions for bubbles to exist are the easier fulfilled the lower the expected return to investment in real capital and the higher is the risk premium demanded on investment in real capital. This finding corresponds well to the literature (Bertocchi, 1991; Bertocchi and Kehagias, 1995; Blanchard and Weil, 2001), which establishes an insurance role for deterministic bubbles (or rolled-over debt), which is the more important, the riskier is investment in real capital.

The interesting cases arise when $\log \left(1+r^{d}(A)\right)$ fluctuates above and below $\log (1+n)$ randomly. Then the current value of the marginal product of capital cannot be used as an indicator in order to determine whether bubbles may exist. It is the expected path of future states (which investors infer from the observed history of past states) that determines dynamic efficiency and the possible existence of bubbles in the stochastic economy. This fact has important implications for empirical research on dynamic efficiency as will be shown in the following sections.

\section{Simulation of a Bubble in a Stochastic Economy with Logarithmic Utility and Cobb-Douglas Production Function}

So far we have established conditions under which bubbles may exist in a stationary stochastic economy and we have shown that they are able to cure overaccumulation of capital. However, we have not discussed the stability properties of the stationary equilibria with a positively valued bubble. According to Bertocchi (1994), stationary states with a deterministic bubble can be characterized as a stable set ${ }^{12}$, where the capital stock $k_{t}$ once it enters an interval $\left[k_{\min }, k_{\max }\right]$ will never leave it thereafter. However, a stable set does not exist, provided that saving is a nondecreasing function of the interest rate (Bertocchi, 1994, p. 504). This result would imply that bubbles are not able to permanently cure economies from capital overaccumulation (Bertocchi, 1994, p. 506). Therefore, the question arises: Do bubbles still have a role to play in stochastic economies?

12 See Wang (1993, p. 428) for a definition. 
In this section, we will present a simulation of a stationary stochastic "bubble economy" showing that, if we assume specific utility and production functions, stationary bubbles can exist for extended periods under plausible conditions in spite of the eventual instability of the resulting stationary state. As soon as we describe actual economies and leave our highly stylized model world, dynamic efficiency can only be assessed by looking at the finite history of a specific economy. ${ }^{13}$ And from the relatively short history that economic agents are actually able to observe, they are generally unable to infer the stability properties, which have been established for overlapping generations economies that exist for an infinite period of time. Consequently, what matters in the real world is dynamic efficiency as it is perceived over a finite period.

Given the existence of a temporary stationary equilibrium over a finite number of periods, rational economic agents have no other choice than to base their expectations of the future development on this particular history of the economy and to assume that the equilibrium will also prevail in the future. This is due to the fact that from the observed history it is impossible to infer whether the bubble will remain stationary, will eventually explode, or will converge towards zero. Over finite periods, bubbles can still be considered to be welfare increasing even if, over an infinite time period, they are not. But the infinite time scale is beyond the scope of actual decision making of economic agents.

Consider an economy with a logarithmic utility function and a CobbDouglas production function with multiplicative shocks $A_{t}$, i.e., $u\left(c_{t}\right)=\log \left(c_{t}\right)$ and $y_{t}=A_{t} k_{t}^{\rho}-\delta k_{t}$, where $\delta$ stands for the depreciation rate. ${ }^{14}$ Based on these assumptions the dynamics of a bubbleless economy characterized by (5), (6), with $P_{t}=P_{t+1}=0$, and (12) are expressed by the first-order, nonlinear difference equation in $k_{t}^{d}$

$$
k_{t+1}^{d}=\frac{(1-\rho) A_{t}\left(k_{t}^{d}\right)^{\rho}}{(2+\phi)(1+n)} .
$$

13 This point is also highlighted by Zilcha (1991, p. 6), and Barbie et al. (2004). It is also very common to use the relatively short history of stock prices to assess expected returns and risk premia on the stock market because this is the only information available to investors.

14 A logarithmic utility function is assumed because otherwise the first-order conditions (5), (6) cannot be solved analytically. 
If there is a bubble, the dynamics of the economy characterized by (5), (6), (11) and (12) can be expressed by a system of two first-order, nonlinear difference equations in $\left(k_{t}, b_{t}\right)$ :

$$
\begin{aligned}
k_{t+1}= & \frac{\frac{(1-\rho) A_{t} k_{t}^{\rho}}{(2+\phi)}-b_{t}}{1+n}, \\
\frac{b_{t+1}}{b_{t}}(1+n)= & 1+\left(1+\frac{h z\left(1+\left(A_{L} \rho k_{t+1}^{\rho-1}-\delta\right)\right)}{(1-z)\left(1+(1+h)\left(A_{L} \rho k_{t+1}^{\rho-1}-\delta\right)\right)}\right) \\
& \times\left(A_{L} \rho k_{t+1}^{\rho-1}-\delta\right) .
\end{aligned}
$$

According to Blanchard and Fischer (1989, p. 147) the following parameter values for $n, \delta$ and $\phi$ are plausible for a period of about 30 years and they have to be altered if the model is applied to shorter time scales. Specifically we assume: $\rho=0.25, A_{L}=20, A_{H}=22$ (therefore, $h$ $=0.1), z=0.5, n=0.35, \delta=1, \phi=0.85$. The simulation is done for one hundred periods and the values for $A_{t}$ are generated by using a random number generator to pick the values 20 and 22 with equal probability. The simulations are displayed in Fig. 1.

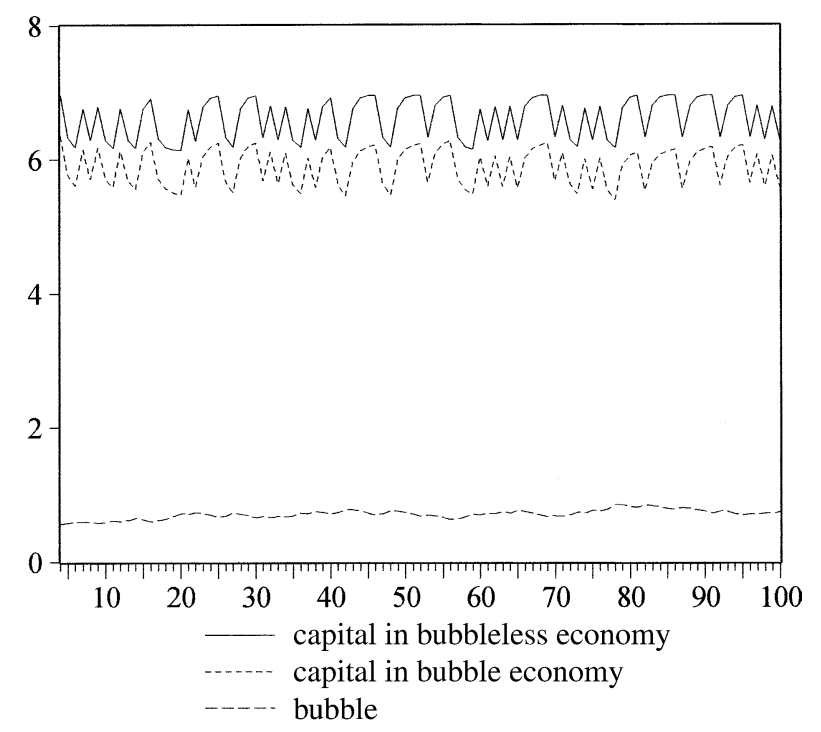

Fig. 1. Simulation of a stochastic economy without a bubble compared to a simulation of a stochastic economy with a bubble over 100 periods. The figure shows the per capita levels of capital and the bubble as calculated by Eqs. (24)-(25) 
First, we simulate a bubbleless economy described by (23) starting with an initial value of $k_{0}^{\mathrm{d}}=7$ in the first simulation period. However, if we start with different initial values $\left(k_{0}^{\mathrm{d}}>0\right)$, capital stocks quickly converge to the same path $k_{t}^{d *}$ after a few periods for a given sequence of $A_{t}$. In our example, the capital stock $k_{t}^{d *}$ fluctuates between values of 6.14 and 6.97 while the marginal product $r^{d *}\left(A_{t+1}, T^{t} A\right)$ fluctuates between values of 0.16 and 0.41 . It can be shown that the interval $\left[k_{\min }^{\mathrm{d}}, k_{\max }^{\mathrm{d}}\right]=[6.14,6.97]$ is a stable set, which, because of our assumptions of a logarithmic utility function and of a Cobb-Douglas production function, also exhibits global uniqueness and stability for $k_{0}^{\mathrm{d}}>0$ (Wang, 1993 , p. 433). Once the economy enters the interval $\left[k_{\min }^{\mathrm{d}}, k_{\max }^{\mathrm{d}}\right]$ it stays there forever. Moreover, using random dynamical systems theory, $k_{t}^{d *}$ can be described as a globally attracting random fixed point (Peter and Schenk-Hoppé, 1999; Schenk-Hoppé and Schmalfuss, 2001).

If we assume that agents know the particular history of the economy for the simulated time span of 100 periods, they can only base their expectations on these past observations and the expected value $E\left[r^{d}(A)\right]$ is the mean of the 100 observed values. Observing the history of our simulated bubbleless economy, agents will calculate that $E\left[\log \left(1+r^{d}(A)\right]\right.$ is equal to 0.11 while $\log (1+n)$ equals 0.13 . Therefore, the economy is in a dynamically inefficient state where the average lifetime consumption of an agent is 26.83. However, the dynamic inefficiency cannot be inferred from observing the current state of the economy because $1+r^{d}\left(T^{t} A\right)$ fluctuates between values that are below and above $1+n$.

Second, we simulate an economy with a temporarily stationary deterministic bubble described by the system (24), (25). We use the same random numbers as in the bubbleless economy starting with initial values of $k_{0}=7$ and $b_{0} \cong 0.6761$. In this case, the system is very sensitive to the choice of the initial values $k_{0}, b_{0}$. Given $k_{0}=7$, and the particular sequence of random numbers picked for the simulation, there is only a very small range of initial values around 0.6761 that leads to a stationary bubble over 100 periods. Otherwise the bubble will either explode (if $b_{0}>0.6761$ ) or converge towards zero (if $b<0.6761$ ) within this time span. ${ }^{15}$

15 A different sequence of random numbers would of course require a different initial value $b_{0}$ for the bubble to be stationary over the time span of 100 periods. I have simulated various bubble economies described by the system (24), (25) with different parameter values and it is always possible to find bubbles that are stationary for the simulated time span of 100 periods. 
In the simulated example, the capital stock $k_{t}$ now fluctuates between values of 5.42 and 6.30 after a few periods and is, therefore, on a lower level than in the bubbleless economy. The marginal product fluctuates between values of 0.26 and 0.53 and $E[\log (1+r(\mathrm{~A}))]$ equals 0.14 . Therefore, the bubble economy is temporarily dynamically efficient because $\log (1+n)<E[\log (1+\mathrm{r}(A))]$ and the average lifetime consumption is now 27.13 as compared to 26.83 in the bubbleless economy. The bubble itself fluctuates between values of 0.57 and 0.82 . The simulation provides an example where a stationary bubble is able to temporarily remove the dynamic inefficiency in a stochastic economy and is, therefore, welfare increasing over the simulated time periods. Even in a period $t$ where $1+r\left(T^{t} A\right)>1+n$ a bubble may exist and it keeps the expected return to capital at a level that is dynamically efficient.

The analysis in Bertocchi (1994, p. 504) shows that the stationary equilibrium in the corresponding deterministic economy with logartihmic utility and Cobb-Douglas production function would be a saddlepoint. But a stable set does not exist for the stochastic economy, which rules out the existence of a stochastic stationary state. However, as the simulated pattern of the stochastic economy with a bubble shows, the economy still appears to be stationary for the simulated time span. Therefore, agents may rationally assume that the stationary set will prevail in the future, because agents just know the particular history of the economy over the simulated 100 periods. This is the important message from our simulation exercise.

In reality the potential of bubbles to cure overaccumulation of capital will also hinge on factors that contribute to the sustainability of bubbles. In this section and Sect. 4, sustainability was assumed by limiting the analysis to deterministic bubbles. But in reality investors' confidence in the future survival of a bubble will be a crucial factor in this respect. Bubbles probably also need to be "managed" in order to be sustainable. If, for example, the central bank issues warnings that stock prices are overvalued when bubbles are in danger of exploding and, on the other hand, emphasizes the strength of the economy when bubbles are in danger of bursting or converging towards zero, bubbles may become more sustainable. Therefore, it would also be useful to develop models that integrate behavioral explanations of investors' confidence as they may substantially alter the stability properties of bubble equilibria. A first step in this direction is Bertocchi and Wang (1995) who endogenize the probability that the bubble collapses by linking it to past observations. 


\section{Empirical Evidence on Dynamic Efficiency and the Existence of Bubbles}

Existing empirical research appears to indicate that the US economy as well as the economies in other industrialized countries are dynamically efficient. Cohen et al. (1995) infer the marginal product of capital from capital's share of GDP and calculate golden rule levels of the capital stock and of net investment as a percentage of GDP, which they compare to the actual levels in the US over the period from 1980 to 1994. According to their results the actual levels of the capital stock and of net investment are below the golden rule levels and, therefore, the US economy would be dynamically efficient. More recently, Mulligan (2001) provides a series of pre-direct tax return to capital (capital rental rates) in the US, where the return to real capital is capital income net of depreciation as a percentage of the capital stock. On average, the return to capital was 8.3 percent over the period from 1929 to 1997 and fluctuated between values of 3.7 percent and 12.5 percent. Taking the 8.3 percent calculated by Mulligan (2001) and using the dynamic efficiency criterion derived by Zilcha (1991), Barbie et al. $(2001 ; 2004)$ find the US economy to be dynamically efficient from 1929 till 1997 and 1890 till 1999, respectively.

Assessing dynamic efficiency by using aggregate measures of the capital stock can be subject to severe measurement errors. This difficulty is avoided by the net cash flow criterion developed by Abel et al. (1989), which states that an economy is dynamically efficient as long as gross profits exceed gross investment in every year. This (sufficient) criterion also seems to be fulfilled for the US over the period from 1929 to 1985 and for other OECD countries from 1960 to 1984 as shown in Abel et al. (1989), although the result has been criticized on various grounds (Anderson, 1993; Bullard and Russell, 1999; Barbie et al., 2004).

Given the empirical evidence in favor of dynamic efficiency of the US economy and other OECD countries, it has usually been argued that stationary bubbles cannot exist in these economies as dynamic efficiency rules out the existence of bubbles. However, based on the simulation presented in this paper, this conclusion may be questioned for a reason, that has not been mentioned in the literature so far. Empirical research on dynamic efficiency can hardly distinguish between an economy that is dynamically efficient without a bubble and an economy that is dynamically efficient due to the existence of a bubble. Bubbles are hard to identify in stochastic economies where uncertainty about the future 
development of the economy is a pervasive phenomenon and where the market fundamentals depend on unobservable expectations. Therefore, empirical reasearch which indicates dynamic efficiency of existing economies may not be used as an argument to rule out the existence of bubbles in these economies.

Relating our model to the more recent history of the US economy over the 1980s and 1990s, there is some evidence that bubbles could indeed have helped to keep the economy in a dynamically efficient state. If markets are characterized by excess capacities and saturation as seemed to be the case in several sectors of the US economy during the 1980 s (Jensen, 1988; 1993), the return to capital tends to be low. In the early 1980 s, many investors reacted to the low returns to real capital by investing in already existing companies (mergers and acquisitions wave) instead of investing in new real capital (see Jensen, 1993). This change in investment behavior caused stock prices to surge, while gross investment in real capital as a share of GDP came down over the 1980s. The unprecedented rise in stock prices over the 1980s and 1990s can be interpreted as a speculative bubble (see, for example, Binswanger, 1999; Shiller, 2000) that helped to restore the return to real capital above its low level in the early 1980s. Even if empirical research indicates dynamic efficiency by looking at data from these years it may be the result of an existing bubble that prevented the economy from overaccumulation of real capital. Under these circumstances dynamic efficiency would not rule out the existence of a bubble. On the contrary, the result would be the outcome of an already existing bubble.

\section{Conclusion}

In this paper we presented a simple model where the economy fluctuates between two states of nature captured by the level of productivity and highlighted conditions under which bubbles may exist in this economy. It turns out that under these conditions dynamic inefficiency is not a necessary condition for the existence of bubbles and a stationary bubble may even exist in a dynamically efficient economy. Generally, given the growth rate of population, the conditions for bubbles to exist are the easier fulfilled the lower is the expected return to investment in real capital and the higher is the volatility of these returns.

However, the theoretical results derived from the model presented in Sects. 3 and 4 cannot provide an answer to the important question 
whether the welfare increasing potential of sustainable bubbles in dynamically inefficient economies is of any relevance to actual economies, as there exist theoretical and empirical arguments that question the feasibility of curing these economies from capital overaccumulation. Theoretical models (Bertocchi, 1994) suggest that, even if economies were dynamically inefficient, the resulting equilibria would eventually be unstable. And empirical results indicate that the US economy as well as the economies in other industrialized countries are dynamically efficient.

In order to deal with these arguments we also presented a simulation of a stationary stochastic bubble economy showing that bubbles can indeed exist over long periods and that they are able to increase economic welfare. Bubbles may also prevent stochastic economies from capital overaccumulation and, therefore, this important welfare increasing potential of bubbles, which was derived for deterministic economies by Tirole (1985), may also be valid for actual economies. Furthermore, the simulation also shows that an economy may exhibit dynamic efficiency precisely because bubbles already exist. Consequently, empirical results based on current or past data of existing economies indicating dynamic efficiency (see, for example, Abel et al., 1989; Barbie et al., 2001; 2004; Cohen et al., 1995) cannot generally be used to rule out the existence of bubbles.

Finally, we have to emphasize some of the aspects that could not be dealt with in the simple model presented in this paper, which however are important. Assessing dynamic efficiency by using the criterion developed by Zilcha (1991) was only possible by restricting the analysis to stationary deterministic bubbles, which are sustainable in the long run. Therefore, a characterization of the dynamics of a stochastic bubble in a stochastic economy is still missing and it is still unclear how the results would translate into a nonstationary economy. ${ }^{16}$ Furthermore, the paper also shows the limits of models built on rational expectations to deal with phenomena such as speculative bubbles. Therefore, the integration of behavioral decision theory in these models may be a promising direction for future research.

\section{Acknowledgements}

The author would like to thank Klaus Rainer Schenk-Hoppé and three anonymous referees for very helpful suggestions and comments.

16 Tools for analyzing this class of models in nonstationary environments are still in the process of being developed (Schenk-Hoppé, 2001). 


\section{References}

Abel, A., Mankiw, G., Summers, L., and Zeckhauser, R. (1989): “Assessing Dynamic Efficieny: Theory and Evidence." Review of Economic Studies 56: $1-20$.

Anderson, G. (1993): “An Empirical Note on Assessing Dynamic Efficiency: Integration, Co-integration, and the Long Run." Structural Change and Economic Dynamics 4: 345-352.

Barbie, M., Hagedorn, M., and Kaul, A. (2001): Government Debt as Insurance Against Macroeconomic Risk. IZA Discussion Paper No. 412, Bonn.

Barbie, M., Hagedorn, M., and Kaul, A. (2004): “Assessing Aggregate Tests of Efficiency for Dynamic Economies". Working Paper, University of Karlsruhe.

Bertocchi, G. (1991): "Bubbles and Inefficiencies." Economics Letters 35: 117122.

Bertocchi, G. (1994): “Safe Debt, Risky Capital.” Economica 61: 493-508.

Bertocchi, G., and Kehagias, A. (1995): "Efficiency and Optimality in Stochastic Models with Production." Journal of Economic Dynamics and Control 19: 303-325.

Bertocchi, G., and Wang, Y. (1995): “The Real Value of Money under Endogenous Beliefs." Journal of Economic Theory 67: 205-222.

Binswanger, M. (1999): Stock Markets, Speculative Bubbles and Economic Growth. Cheltenham: Edward Elgar.

Blanchard, O. (1979): "Speculative Bubbles, Crashes and Rational Expectations." Economics Letters 3: 387-389.

Blanchard, O., and Watson, M. (1982): "Bubbles, Rational Expectations and Financial Markets." In Crises in the Economic and Financial Structure, edited by Paul Wachtel. Lexington, MA: Lexington Books.

Blanchard, O., and Fischer, S. (1989): Lectures on Macroeconomics. Cambridge, MA: MIT-Press.

Blanchard, O., and Weil, P. (2001): "Dynamic Efficiency, the Riskless Rate and Debt Ponzi Games under Uncertainty." Advances in Macroeconomics 1(2), Article 3.

Breiman, L. (1968): Probability. Reading, MA: Addison-Wesley.

Bullard, J., and Russell, S. (1999): "An Empirically Plausible Model of Low Real Interest Rates and Unbacked Government Debt." Journal of Monetary Economics 44: 477-508.

Caballero, R., and Hammour, M. (2002): "Speculative Growth.” NBER Working Paper No. 9381. Cambridge, MA.

Cass, D. (1972): “On Capital Overaccumulation in the Aggregative Neoclassical Model of Economic Growth: A Complete Characterization." Journal of Economic Theory 4: 200-223.

Cohen, D., Hasset K., and Kennedy, J. (1995): “Are U.S. Investment and Capital Stocks at their Optimal Levels?," FEDS Working Paper No. 9532. Board of Governors of the Federal Reserve System.

Diamond, P. (1965): "National Debt in a Neoclassical Growth Model." American Economic Review 55: 1126-1150.

Jensen, M. (1988): "The Takeover Controversy: Analysis and Evidence." In Knights, Riders and Targets, edited by J. Coffee. Oxford University Press. 
Jensen, M. (1993): “The Modern Industrial Revolution, Exit and the Failure of Internal Control Systems." Journal of Finance 48: 831-880.

Mulligan, C. (2001): "Capital, Interest, and Aggregate Intertemporal Substitution." Working Paper No. 9373. National Bureau of Economic Research, Cambridge, MA.

Olivier, J. (2000): “Growth-enhancing Bubbles.” International Economic Review 41: 133-151.

Peter, J. P., and Schenk-Hoppé, K. R. (1999): "Business Cycle Phenomena in Overlapping Generations Economies with Stochastic Production." Working Paper No. 30. Institute for Empirical Reasearch in Economics, University of Zurich.

Schenk-Hoppé, K. R. (2001): "Random Dynamical Systems in Economics." Stochastics and Dynamics 1: 63-83.

Schenk-Hoppé, K. R., and Schmalfuss, B. (2001): "Random Fixed Points in a Stochastic Slow Growth Model." Journal of Mathematical Economics 36: 1930 .

Shiller, R. (2000): Irrational Exuberance. Princeton University Press.

Tirole, J. (1985): “Asset Bubbles and Overlapping Generations." Econometrica 53: $1499-1528$.

Wang, Y. (1993): "Stationary Equilibria in an Overlapping Generations Economy with Stochastic Production." Journal of Economic Theory 61: 423-435.

Weil, P. (1987): "Confidence and the Real Value of Money in an Overlapping Generations Economy." Quarterly Journal of Economics 102: 1-22.

Zilcha, I. (1990): "Dynamic Efficiency in Overlapping Generations Models with Stochastic Production." Journal of Economic Theory 52: 364-379.

Zilcha, I. (1991): "Characterizing Dynamic Efficiency in Stochastic Overlapping Generations Models.” Journal of Economic Theory 55: 1-16.

Addresses of author: - M. Binswanger, University of Applied Sciences of Northwestern Switzerland, Riggenbachstr 16, 4600 Olten, Switzerland (e-mail: mathias.binswanger@fhso.ch) and University of St. Gallen, 9000 St. Gallen, Switzerland (e-mail: mathias.binswanger@unisg.ch) 